'Sección de Medicina de Urgencia, División de Medicina, Facultad de Medicina, Pontificia Universidad Católica de Chile. ${ }^{2}$ Centro de informática Biomédica, instituto de ciencias e innovación en medicina, Facultad de Medicina Clínica Alemana Universidad del Desarrollo, Universidad del Desarrollo. alnternos de Medicina, Universidad de los Andes. bEstudiante de Medicina, University of Southern California.

Recibido el 14 de julio de 2017 aceptado el 24 de octubre de 2017

Los autores no declaran conflictos de interés

Trabajo no recibió financiamiento

Correspondencia a: Dra. Bárbara Lara balara@uc.cl

\section{Paro cardiorrespiratorio extrahospitalario. Realidad de un hospital terciario chileno}

\author{
BÁRBARA LARA ${ }^{1}$, MARÍA JOSÉ VALDÉS ${ }^{\mathrm{a}}$, \\ RAIMUNDO SAAVEDRA ${ }^{a}$, JOSÉ VARGAS ${ }^{1}$, JOAQUÍN CHUECAS ${ }^{1}$, \\ CRISTHOFER OPAZO ${ }^{1}$, EMILY NEIL ${ }^{\mathrm{b}}$, MARCELO LOPETEGUI ${ }^{2}$, \\ DAVID ACUÑA ${ }^{1}$, PABLO AGUILERA ${ }^{1}$
}

\section{Out of hospital cardiac arrest events at an urban Hospital in Chile}

Background: The incidence of out of hospital cardiac arrest (OHCA) is approximately 20 to 140 per 100.000 inhabitants. International registries, based on Utstein criteria have allowed standardized reporting of OHCA profiles and outcomes in different countries. We proposed to create a local OHCA registry. Aim: To assess the quality of the information about OHCA currently recorded in medical records according to Utstein guidelines. Material and Methods: A retrospective analysis of medical records of patients arriving in the emergency room of a public hospital with OHCA during a 3-year period. Data regarding the patient characteristics, event and outcomes were analyzed. Results: During the revision period, 317 patients arrived with an OHCA. None of the medical records had complete data on items that are considered a minimum requirement by Utstein guidelines. Mean age of patients was 63 years old, 60\% were men, the most common arrest rhythm was asystole (43\%) and $8 \%$ of patients were discharged alive. Conclusions: Data recorded in medical records is insufficient to inform the profile of OHCA. A prospective registry is currently being implemented based on the information provided by this study. This registry should optimize reporting and data analysis.

(Rev Med Chile 2017; 145: 1308-1311)

Key words: Chile; Hospitals, Public; Out-of-Hospital Cardiac Arrest; Registries.
E l paro cardiorrespiratorio extra-hospitalario (PCR-EH) se define como el cese abrupto de la actividad mecánica cardíaca, confirmado por la ausencia de signos circulatorios, que ocurre de manera inesperada, fuera de un recinto hospitalario ${ }^{1}$.

Hace más de un siglo se reconoció la falta de acuerdo en la definición de paro cardiorrespiratorio, lo que dificultaba la estandarización en su manejo. Así, en 1992 se formó un comité internacional de resucitación (ILCOR) con el fin de unificar definiciones, procedimientos de re- gistro y manejo del PCR. Desde entonces, se han creado guías de reporte y recolección de datos de PCR, conocidas en la literatura como criterios de Utstein ${ }^{1}$. A partir de esas guías, se han generado una variedad de registros de PCR-EH de distinta envergadura y perfiles epidemiológicos ${ }^{2-6}$.

A pesar de que la recolección de datos es el primer paso clave para determinar la efectividad de estrategias terapéuticas y poder comparar su utilidad en distintas poblaciones, en Chile no se ha creado un registro que permita reportar datos de manera estandarizada ${ }^{7}$. El único reporte de 
PCR-EH en Chile es el publicado por Mayans et $\mathrm{al}$, en donde se describe una cohorte de pacientes atendidos por el SAMU metropolitano (servicio de ambulancias de urgencia $)^{8}$.

Para suplir esa falencia -previo a la creación de un registro prospectivo- planificamos este proyecto que tiene por objetivo evaluar la cantidad y calidad de la información registrada en las fichas clínicas respecto al PCR-EH y el cumplimiento de registro de datos mínimos requeridos según las recomendaciones de la ILCOR y reportar el perfil de PCR-EH en un centro terciario en base a estos datos.

\section{Materiales y Métodos}

El estudio se llevó a cabo en el Complejo Asistencial Sótero del Río (CASR), un hospital urbano de alta complejidad que cuenta con un total de 754 camas, su población asignada corresponde a $9 \%$ de la población chilena y atiende a 150.000 pacientes en el servicio de urgencia (SU) al año9.

Se realizó una revisión retrospectiva de todas las fichas de las consultas de urgencia con categoría de Triage $\mathrm{C} 1$ en un período de casi 3 años ${ }^{10}$. Se incluyó en el análisis las atenciones de pacientes que ingresaron al SU por PCR-EH independiente de su método de traslado al SU o de si lograron o no retorno a circulación espontánea previo a la atención. Se excluyeron atenciones de pacientes menores de 18 años y a aquellos en que la falta de registro imposibilitara la validación de dicha atención.

Se recolectaron los datos sugeridos y catalogados como mínimos por la las guías de la ILCOR 2015 respecto a los pacientes, el evento, $\mathrm{y}$ el desenlace ${ }^{1}$.

Definimos como PCR-EH presenciado, a eventos en que testigos presenciaron el momento exacto de la perdida de tono y/esfuerzo respiratorio, independiente de haber o no iniciado maniobras de reanimación. Definimos "sobrevida al evento" lograr retorno a circulación espontánea (RCE) sostenida. Dado que no se realizaban epicrisis electrónicas en cuidados intensivos o en la urgencia durante el período estudiado, para el desenlace compuesto "sobrevida al alta o a 30 días" se consideraron vivos a todos los pacientes que: a) tenían una epicrisis disponible y fueron dados de alta vivos independiente del tiempo al alta b) no tenían epicrisis disponible y la fecha muerte repor- tada en el certificado de defunción fue extendido en el CASR y la fecha de muerte fue posterior al día 30 desde el PCR-EH. Aquellos pacientes con egreso hospitalario administrativo, sin epicrisis y sin certificado de defunción a los 30 días y al año del evento, se consideraron vivos a 30 días y al año respectivamente.

\section{Resultados}

Entre 1 de enero de 2013 y al 30 de noviembre de 2015 hubo 393.714 consultas al servicio de urgencia del CASR. Un $0,7 \%(\mathrm{n}=2.756)$ fueron categorizados como $\mathrm{C} 1$ y revisadas para identificar los casos de PCR-EH. Un total de 317 cumplían con la definición de PCR-EH y criterios de inclusión y exclusión.

Ningún dato de atención y ficha clínica cumplió con el registro de toda la información recomendada por el Comité de ILCOR 2015 (Tabla 1).

\section{Tabla 1. Disponibilidad de información catalogada como mínima de acuerdo a criterios de ILCOR}

\begin{tabular}{|lrr|}
\hline & $\begin{array}{c}\text { n dispo- } \\
\text { nibles }\end{array}$ & $\begin{array}{c}\text { \% del } \\
\text { total }\end{array}$ \\
Paciente & 317 & 100 \\
Edad & 317 & 100 \\
Sexo & 90 & 28 \\
PCR-EH presenciado por testigos & 54 & 17 \\
RCP por testigos & 61 & 19 \\
Lugar del PCR & 10 & 3 \\
Uso de DEA & 222 & 70 \\
Ritmo inicial & 60 & 19 \\
Etiología del PCR & & \\
Proceso & 0 & 0 \\
Tiempo de respuesta & 0 & 0 \\
Tiempo a desfibrilación & 0 & 0 \\
Uso de hipotermia & 0 & 0 \\
Coronariografía & & \\
Desenlaces & 317 & 100 \\
Sobrevida al evento* & 315 & 99 \\
Sobrevida al alta o 30 días & 0 & 0 \\
Pronóstico neurológico & & \\
\hline
\end{tabular}

No se incluyeron en esta tabla datos referentes a la población, indicaciones por regulador de ambulancias, ni elementos que son considerados opcionales o adicionales según Utstein. *Sobrevida al evento: mantiene retorno a circulación espontánea (RCE) hasta el CASR si fue trasladado tras RCP o mantiene RCE por más de una hora si ingresa en PCR al CASR.DEA: desfibrilador externo automático.PCR-EH: Paro cardiorrespiratorio extra hospitalario. 
Variables epidemiológicas de los que se disponía información respecto de los pacientes y del evento se encuentra detallados en la Tabla 2. La sobrevida al alta o a 30 días fue de $8 \%$ (Figura 1). Se desconoce el desenlace neurológico de dichos pacientes.

\section{Discusión}

Los resultados de este estudio dejan en manifiesto la carencia de información relevante referente a eventos de PCR-EH que se registra en las fichas de manera habitual, y la necesidad de tener un registro de PCR protocolizado.

La definición de sobrevida al alta o a 30 días usada en este estudio, se basa fundamentalmente en la convicción de que los certificados de defunción proveen información certera respecto a la ocurrencia, fecha y lugar del desenlace "mortalidad". Considerando esta limitación, la estimación de la tasa de sobrevida en este estudio es similar a las reportada por países desarrollados en PCR$\mathrm{EH}$ indiferenciados $(8 \%)^{3,5,11,12}$. Por el contrario, esta cifra es significativamente mayor a la reportada por la serie de pacientes reanimados por el SAMU de Mayanz et al $(3,3 \%)^{8}$. Las diferencias en sobrevida probablemente se expliquen por las diferencias en el perfil de las poblaciones estudiadas, la capacidad de cuidados intra y post paro
Tabla 2. Características del PCR en base a información disponible

\begin{tabular}{|lrr|}
\hline Características del PCR & $\begin{array}{c}\text { Denomi- } \\
\text { nador* }\end{array}$ & n (\%) \\
Paciente & 317 & $63 \pm 16$ \\
Edad, x \pm DE & 317 & $184(58)$ \\
Hombres & 90 & $43(47)$ \\
PCR presenciados & 54 & $1(1)$ \\
RCP por testigos & & \\
Lugar del PCR & 61 & $46(75)$ \\
Domicilio & 61 & $15(25)$ \\
Vía pública & & \\
Ritmo inicial & 222 & $146(66)$ \\
Asistolia & 222 & $38(17)$ \\
AESP & 222 & $31(14)$ \\
FV & 222 & $7(3)$ \\
TV & & \\
Etiología del PCR & 60 & $21(35)$ \\
Cardíacas & 60 & $39(65)$ \\
No cardíacas & & \\
Desenlaces & 317 & $107(34)$ \\
Sobrevida al evento & 317 & $27(9)$ \\
Sobrevida al alta o 30 días & & \\
\hline *Para eá calculo del porentaje & & \\
\hline
\end{tabular}

* Para el cálculo del porcentaje de pacientes con cierta característica, se usó como denominador el número total de pacientes en que había información disponible. AESP: Actividad eléctrica sin pulso. FV: fibrilación ventricular. TV: taquicardia ventricular.

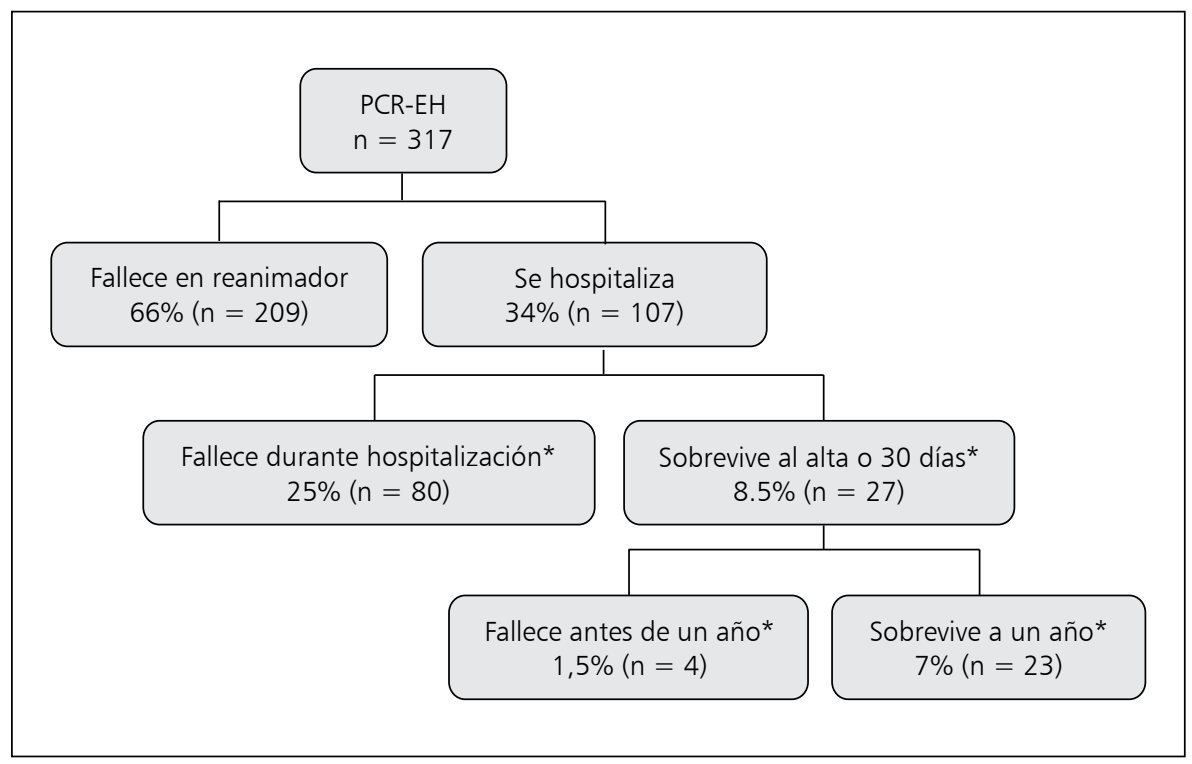

Figura 1. Flujograma de sobrevida al evento y alta hospitalaria. PCREH: paro cardiorespiratorio extrahospitalario. * Sobrevida estimada en base a fechas y lugar de defunción de certificados de defunción. Se desconoce la funcionalidad neurológica de estos pacientes. 
de ambas poblaciones, así como las definiciones de sobrevida al alta. Por otro lado, muchos de los pacientes reanimados por personal del SAMU o SAPU que no logran RCE, no son trasladados, contribuyendo a la selección de pacientes con mayor tasa de sobrevida. Sin embargo, los datos con los que contamos, no permiten hacer más que conjeturas.

Los datos de un registro prospectivo permitirán detallar con mayor precisión el perfil de estos pacientes, y a su vez demostrar la necesidad de educación de la población en soporte vital básico.

Este es el primer estudio chileno que se acerca a describir el perfil epidemiológico de pacientes con PCR-EH atendidos en un centro terciario y ha aportado información fundamental para crear un registro de PCR-EH piloto prospectivo, que ya se encuentra en desarrollo.

\section{Referencias}

1. Perkins GD, Jacobs IG, Nadkarni VM, Berg RA, Bhanji F, Biarent D, et al. Cardiac Arrest and Cardiopulmonary Resuscitation Outcome Reports: Update of the Utstein Resuscitation Registry Templates for Out-of-Hospital Cardiac Arrest A Statement for Healthcare Professionals From a Task Force of the International Liaison Committee. Circulation 2015; 131 (13): 1286-300.

2. Mashiko K, Otsuka T, Shimazaki S, Kohama A, Kamishima G, Katsurada K, et al. An outcome study of out-of-hospital cardiac arrest using the Utstein template-a Japanese experience. Resuscitation 2002; 55 (3): 241-6.

3. Facility H, Place I, Home N, Witnessed B, Applicable N, Bystanders T, et al. CARES Summary Report Demographic and Survival Characteristics of OHCA Demographic and Survival Characteristics of OHCA 2012; 1909 : 11-2.
4. Ong MEH, Shin S Do, Tanaka H, Ma MHM, Khruekarnchana P, Hisamuddin N, et al. Pan-Asian Resuscitation Outcomes Study (PAROS): Rationale, methodology, and implementation. Acad Emerg Med 2011; 18 (8): 890-7.

5. Gräsner JT, Lefering R, Koster RW, Masterson S, Bttiger BW, Herlitz J, et al. EuReCa ONE-27 Nations, ONE Europe, ONE Registry: A prospective one month analysis of out-of-hospital cardiac arrest outcomes in 27 countries in Europe. Resuscitation 2016; 105: 188-95.

6. Beck B, Bray JE, Smith K, Walker T, Grantham H, Hein C, et al. Establishing the Aus-ROC Australian and New Zealand out-of-hospital cardiac arrest Epistry 2016; 5 (6).

7. McNally B. The importance of cardiac arrest registries. Scand J Trauma Resusc Emerg Med 2014; 22 (Suppl 1): A3.

8. Mayanz S, Barreto J, Grove X, Breinbauer H. Paro cardiorrespiratorio extra-hospitalario de causa cardiaca en Santiago de Chile: Experiencia del equipo medicalizado del SAMU Metropolitano. Rev Med Chile 2009; 24 (1): 9-16.

9. Complejo Asistencial Dr. Sótero del Río. http://www. hospitalsoterodelrio.cl/web/quienes-somos/. Accessed May 30, 2017.

10. Acuña Ramírez D, Lara Hernández B, Basaure C, Navea Carrasco O, Kripper Mitrano C, Saldías Peñafiel F. Series clínicas de Medicina de Urgencia. Estratificación de riesgo (Triage) en el Servicio de Urgencia. Rev Chil Med Intensiva 2015; 30 (2): 79-86.

11. Hasegawa K, Tsugawa Y, Camargo CA, Hiraide A, Brown DFM. Regional variability in survival outcomes of out-of-hospital cardiac arrest: The All-Japan Utstein Registry. Resuscitation 2013; 84 (8): 1099-107.

12. Ong MEH, Shin S Do, De Souza NNA, Tanaka H, Nishiuchi T, Song KJ, et al. Outcomes for out-of-hospital cardiac arrests across 7 countries in Asia: The Pan Asian Resuscitation Outcomes Study (PAROS). Resuscitation 2015; 96: 100-8. 